\title{
Clinical usefulness of capnographic monitoring when inserting a feeding tube in critically ill patients: retrospective cohort study
}

Jeong-Am Ryu' ${ }^{1}$, Kyoungjin Choi ${ }^{2}$, Jeong Hoon Yang ${ }^{1,3}$, Dae-Sang Lee ${ }^{1}$, Gee Young Suh 1,4, Kyeongman Jeon ${ }^{1,4}$, Joongbum Cho ${ }^{1}$, Chi Ryang Chung ${ }^{1}$, Insuk Sohn ${ }^{5}$, Kiyoun Kim ${ }^{5}$ and Chi-Min Park ${ }^{1,2^{*}}$

\begin{abstract}
Background: It is not rare for a small-bore feeding tube to be inserted incorrectly into the respiratory system in critically ill patients. Thus, monitoring is necessary to prevent respiratory malplacement of the tube. We investigated the utility of capnographic monitoring to prevent respiratory complications due to feeding tube mispositioning in critically ill patients.

Methods: This study was a pre and post-interventional study, including 445 feeding tube placements events studied retrospectively in the medical and surgical intensive care units of the Samsung Medical Center. We compared outcomes between time periods before and after capnographic monitoring and documented any respiratory complications.

Results: Feeding tubes were inserted in 275 cases without capnographic monitoring. Capnographic monitoring was performed in 170 cases. Sixteen patients (4\%) had respiratory complications of all tube placements. Feeding tube was inserted into the trachea in 11 (2\%) patients and for a pneumothorax in five (1\%) patients. Fourteen cases of respiratory complications were detected in the control group (14/275, 5\%, 10 tracheal insertions and four pneumothoraxes). Two respiratory complications were detected in the capnographic monitoring group $(2 / 170,1 \%$, one tracheal insertion and one pneumothorax). Respiratory complications were detected less frequently in the capnographic monitoring group than that in the control group $(P=0.035)$.
\end{abstract}

Conclusions: Capnographic monitoring is simple, easy to learn, and may be useful to prevent respiratory complications when placing a feeding tube in a critically ill patient.

Keywords: Capnographic monitoring, Feeding tube, Critically ill patient, Pneumothorax

\section{Background}

Small-bore feeding tubes have been used to provide proper nutrition and deliver medications to critically ill patients. Inserting a feeding tube is a routine procedure in many intensive care units (ICUs). However, complications associated with inserting a feeding tube have been reported. A major complication of inserting a feeding

\footnotetext{
* Correspondence: dr99.park@samsung.com

'Department of Critical Care Medicine, Samsung Medical Center,

Sungkyunkwan University School of Medicine, Seoul, Republic of Korea

${ }^{2}$ Department of Surgery, Samsung Medical Center, Sungkyunkwan University

School of Medicine, Seoul, Republic of Korea

Full list of author information is available at the end of the article
}

tube is accidental placement of the tube in the respiratory system, resulting in pneumothorax, hydropneumothorax, bronchopleural fistula, atelectasis, empyema, pneumonitis, or pneumonia. These complications are associated with significant morbidity and mortality [1-4]. However, it is not rare for a small-bore feeding tube to be inserted incorrectly into the respiratory system $[2,5-8]$.

Critically ill patients with an endotracheal tube and tracheotomy may exhibit poor response due to their sedated state. Use of an assistant guidewire may contribute to respiratory complications when inserting a feeding tube [9-11]. These conditions are thought to be 
potential risk factors for tracheobronchial malpositioning of a feeding tube in a critically ill patient [2,9-11].

Several methods have been proposed to prevent tracheal malpositioning of a feeding tube. However, some of the methods are not effective, not cost-effective, or require a specialist to prevent respiratory complications when inserting a feeding tube $[6,7,9]$. Capnographic monitoring may be a more effective to prevent respiratory malpositioning of a feeding tube and is a simple and cost-effective method [3, 6, 12-15]. Several studies have reported that capnography helps to prevent respiratory tract malpositioning and confirm feeding tube position. However, these studies were small pilot studies or demonstrated only fragmentary evidence to support its usefulness [3, 12, 14, 15]. We investigated the usefulness of capnographic monitoring to prevent respiratory complications when inserting a feeding tube in a critically ill patient.

\section{Methods}

This retrospective study was performed in a cohort of patients who underwent insertion of a small-bore feeding tube during hospitalization at the medical or surgical ICUs of Samsung Medical Center (1961-bed, universityaffiliated, tertiary referral hospital in Seoul, South Korea) from August 2014 to January 2015. This study was approved by the Institutional Review Board of Samsung Medical Center (SMC 2015-02-076-001) according to the Declaration of Helsinki on reviewing and publishing information from patient's records. Informed consent was waived because of the retrospective nature of the study.

\section{Patients}

A total of 445 feeding tube placements were studied retrospectively. Eligible subjects were $>18$-years-of-age and required a feeding tube according to the ICU physician during their hospital stay. Capnographic monitoring was performed to prevent respiratory tract malplacement while inserting feeding tubes from December 2014 to January 2015. We compared time periods before and after implementing the capnographic monitoring technique. Capnographic monitoring was not performed when tubes were used from August 2014 to October 2014. We defined the feeding tube placements during this period as the control group. Patients were excluded if feeding tube insertion failed or a chest X-ray was not performed to confirm feeding tube position.

\section{Data collection}

The feeding tubes used in all cases were small-bore, flexible feeding tubes (Covidien Kangaroo, Entriflex ${ }^{\mathrm{Tm}}$ Nasogastric Feeding Tubes, 12 Fr, 60 in; Covidien, Dublin, Ireland) and an assistant guidewire was used. A chest X-ray was always performed to confirm the position of the feeding tube. We defined respiratory complications as feeding tubes that were inserted into the tracheopulmonary system (respiratory malplacement of the tube) or direct/indirect lung injuries during an attempt to insert a feeding tube with complications such as pneumothorax, hydropneumothorax, bronchopleural fistula, atelectasis, empyema, pneumonitis, and pneumonia. An artificial airway was defined as having an endotracheal or tracheal tube in place while the feeding tube was being inserted. The Glasgow Coma Scale (GCS) and the Richmond Agitation Sedation Scale (RASS) were used to check patients' mental status and level of sedation. Delirium was defined when the confusion assessment method for the ICU (CAM-ICU) was positive. All variables were investigated when feeding tubes were inserted, except reason for the ICU admission.

\section{Capnographic monitoring protocol}

Each feeding tube was placed according to the following protocol. Tube insertion was monitored with a capnographic unit (Covidien, Microcap ${ }^{\circ}$ Handheld Capnograph). The capnography unit was calibrated according to the manufacturer's instructions. The capnographic unit was turned on and connected to the side hole of the feeding tube. Carbon dioxide $\left(\mathrm{CO}_{2}\right)$ level and flow waveforms were monitored continuously during tube insertion. Placement within the airway was defined as detecting a capnogram wave or an end-tidal carbon dioxide $\left(\mathrm{ETCO}_{2}\right)$ level $>5 \mathrm{~mm} \mathrm{Hg}$. The feeding tube was inserted via a nasal approach to distance of $30 \mathrm{~cm}$ and then held at the tip of the monitor guidewire to detect $\mathrm{CO}_{2}$ for $>20 \mathrm{~s}$ (first step). The tube insertion procedure was stopped if a $\mathrm{CO}_{2}$ waveform appeared or $\mathrm{ETCO}_{2}$ level was $>5 \mathrm{~mm} \mathrm{Hg}$. Then, the feeding tube was removed and reinsertion was attempted. If $\mathrm{ETCO}_{2}$ was not detected during the first step, the feeding tube was inserted the planned distance, and the physician made certain that $\mathrm{ETCO}_{2}$ was monitored for an additional $20 \mathrm{~s}$ (second step). If the airway became involved, the feeding tube was removed by the physician and retried. Finally, a chest X-ray was performed to confirm feeding tube position.

\section{Statistical analyses}

Data are presented as medians and interquartile ranges (IQRs) for continuous variables and as numbers (percentages) for categorical variables. The data were compared using Mann-Whitney $U$ test for continuous variables and the chi-square or Fisher's exact tests for categorical variables. All tests were twosided, and $P<0.05$ was considered significant. Data were analyzed using IBM SPSS Statistics 20 software (IBM, Armonk, NY, USA). 


\section{Results}

A total of 445 feeding tube placements that took place in either the medical or surgical ICUs were analyzed. Of them, 275 cases (August-October 2014) did not have capnographic monitoring performed during insertion. Capnographic monitoring was implemented in December 2014, and 170 feeding tube insertion cases used capnographic monitoring. The median age of the patients was 67 (range 56-75) years, and 294 patients $(66 \%)$ were male. The most common reason for ICU admission was respiratory failure. The baseline characteristics of the capnographic monitoring and control groups are shown in Table 1. There were a few differences between the two groups. Major reasons for ICU admission and ICU locations were different between the two groups. Mechanical ventilator use was more prevalent in the capnographic monitoring group than that in the control group $(P<0.001)$. GCS was higher in the capnographic monitoring group than that in the control group $(P=0.005)$. Sixteen patients $(4 \%)$ had respiratory complications among all tube placements. Tracheal insertion occurred in $11(2 \%)$ patients and pneumothorax in five (1\%). No complication-induced death occurred in either group. Fourteen control group cases had respiratory complications $(14 / 275,5 \%, 10$ tracheal insertions and four pneumothoraxes). Two cases of respiratory complications occurred in the capnographic monitoring group $(2 / 170,1 \%$, one tracheal insertion and one pneumothorax). Elevated $\mathrm{ETCO}_{2}$ level or capnogram wave was detected in 12 cases in the monitored group (12/170, 7\%), and lung injury (pneumothorax)

Table 1 Baseline characteristics of the capnographic monitoring and control groups

\begin{tabular}{|c|c|c|c|c|}
\hline & Overall $(n=445)$ & Capnographic monitoring group $(n=170)$ & Control group $(n=275)$ & $P$-value \\
\hline Age (years) & $67(56-75)$ & $68(59-75.75)$ & $64(55-75)$ & 0.221 \\
\hline Gender (male) & $294(66)$ & $109(64)$ & $185(67)$ & 0.537 \\
\hline \multicolumn{5}{|l|}{ Major reasons for ICU admission } \\
\hline Respiratory failure & $209(47)$ & $81(48)$ & $128(47)$ & \multirow[t]{7}{*}{0.002} \\
\hline Cardiovascular & $72(16)$ & $17(10)$ & $55(20)$ & \\
\hline Severe sepsis or septic shock & $81(18)$ & $45(26)$ & $36(13)$ & \\
\hline Neurological & $49(11)$ & $15(9)$ & $34(12)$ & \\
\hline Post-operation & $17(4)$ & $4(2)$ & $13(5)$ & \\
\hline Multiple trauma & $8(2)$ & $3(2)$ & $5(2)$ & \\
\hline Other & $9(2)$ & $5(3)$ & $4(1)$ & \\
\hline Use of mechanical ventilator & $332(75)$ & $143(84)$ & $189(69)$ & $<0.001$ \\
\hline Artificial airway & $366(82)$ & $151(89)$ & $215(79)$ & 0.004 \\
\hline Endotracheal tube & $242(54)$ & $103(61)$ & $139(51)$ & \\
\hline Tracheal tube & $124(28)$ & $48(28)$ & $76(28)$ & \\
\hline \multicolumn{5}{|l|}{ Location } \\
\hline MICU & $165(37)$ & $62(36)$ & $103(38)$ & \multirow[t]{6}{*}{0.031} \\
\hline SICU & $12(3)$ & $5(3)$ & $7(3)$ & \\
\hline CCU & $46(10)$ & $10(6)$ & $36(13)$ & \\
\hline Cardiac SICU & $11(2)$ & $2(1)$ & $9(3)$ & \\
\hline Oncology MICU & $118(27)$ & $45(26)$ & $73(27)$ & \\
\hline Oncology SICU & $93(21)$ & $46(27)$ & $47(17)$ & \\
\hline GCS & $13(11-15)$ & $14(12-15)$ & $13(10-15)$ & 0.005 \\
\hline RASS & $0(-2-1)$ & $0(-2-1)$ & $-1(-2-1)$ & 0.717 \\
\hline Delirium & $203(59)$ & $86(63)$ & $117(57)$ & 0.216 \\
\hline Use of sedative drug & $207(47)$ & $88(52)$ & $119(43)$ & 0.096 \\
\hline Respiratory complication & $16(4)$ & $2(1)$ & $14(5)$ & \multirow[t]{3}{*}{0.031} \\
\hline Tracheal insertion & $11(3)$ & $1(1)$ & $10(4)$ & \\
\hline Pneumothorax & $5(1)$ & $1(1)$ & $4(2)$ & \\
\hline
\end{tabular}


was reported in only one case. False negative capnographic monitoring occurred in $1 \%$ of cases (tracheal insertion of feeding tube, but $\mathrm{CO}_{2}$ was not detected during one tube placements). No differences were in the demographics detected between the groups with and without respiratory complications in a univariate analysis, except sex (Table 2). More respiratory complications occurred in male patients. Respiratory complications were detected less frequently in the capnographic monitoring group than that in the control group $(P=0.035)$. Respiratory complications were not associated with age, level of consciousness, mechanical ventilator use, artificial airway, or use of a sedative drug in this study (Table 2).

\section{Discussion}

We evaluated the usefulness of capnographic monitoring for preventing respiratory complications and the clinical characteristics associated with inserting a feeding tube in a critically ill patient. The results indicate that capnographic monitoring was useful to prevent respiratory complications while inserting a feeding tube in a critically ill patient. Respiratory complications were not associated with altered mental status, artificial airway, or use of sedative drugs in this study. Respiratory complication rates in all-comers were $4 \%$ in this study. Thus, it is not rare for a small-bore feeding tube to be inserted incorrectly into the respiratory system in a critically ill patient.

Malplacement of a feeding tube in the respiratory tract can lead to various complications associated with significant morbidity and mortality [9]. Several studies have reported that small-bore feeding tubes are inserted into the tracheopulmonary system in $0.3-15.0 \%$ of placement attempts [2, 5-8]. Overall malpositioning-induced complications, such as pneumothorax and hemothorax, occur $(0.7-1.2 \%)$ and complication-induced death in $0.3 \%$ of cases [2, 6, 7]. Most of the patients who

Table 2 Comparisons between the respiratory complication and non-complication groups

\begin{tabular}{|c|c|c|c|c|}
\hline & Overall $(n=445)$ & Respiratory complication group $(n=16)$ & Non-complication group $(n=429)$ & $P$-value \\
\hline Age (years) & $67(56-75)$ & $69(53-80)$ & $67(56-75)$ & 0.669 \\
\hline Gender (male) & $294(66)$ & $15(94)$ & $279(65)$ & 0.015 \\
\hline \multicolumn{5}{|l|}{ Major reasons for ICU admission } \\
\hline Respiratory failure & $209(47)$ & $9(56)$ & $200(47)$ & \multirow[t]{7}{*}{0.493} \\
\hline Cardiovascular & $72(16)$ & $3(19)$ & $69(16)$ & \\
\hline Severe sepsis or septic shock & $81(18)$ & $1(6)$ & $80(19)$ & \\
\hline Neurological & $49(11)$ & $1(6)$ & $48(11)$ & \\
\hline Post-operation & $17(4)$ & $1(6)$ & $16(4)$ & \\
\hline Multiple trauma & $8(2)$ & $0(0)$ & $8(2)$ & \\
\hline Other & $9(2)$ & $1(6)$ & $8(2)$ & \\
\hline Use of mechanical ventilator & $332(75)$ & $14(88)$ & $318(74)$ & 0.379 \\
\hline Artificial airway & $366(82)$ & $15(94)$ & $351(82)$ & \multirow[t]{3}{*}{0.326} \\
\hline Endotracheal tube & $250(55)$ & $111(60)$ & $139(51)$ & \\
\hline Tracheal tube & $129(28)$ & $53(29)$ & $76(28)$ & \\
\hline \multicolumn{5}{|l|}{ Location } \\
\hline MICU & $165(37)$ & $8(50)$ & $157(37)$ & \multirow[t]{6}{*}{0.706} \\
\hline $\mathrm{SICU}$ & $12(3)$ & $1(6)$ & $11(3)$ & \\
\hline $\mathrm{CCU}$ & $46(10)$ & $1(6)$ & $45(10)$ & \\
\hline Cardiac SICU & $11(2)$ & $0(0)$ & $11(3)$ & \\
\hline Oncology MICU & $118(27)$ & $3(19)$ & $115(27)$ & \\
\hline Oncology SICU & $93(21)$ & $3(19)$ & $90(21)$ & \\
\hline GCS & $13(11-15)$ & $14(12-15)$ & $13(11-15)$ & 0.915 \\
\hline RASS & $0(-2-1)$ & $1(-1-1.3)$ & $0(-2-1)$ & 0.153 \\
\hline Delirium & $203(59)$ & $8(62)$ & $195(59)$ & 0.860 \\
\hline Use of sedative drug & $207(47)$ & $10(63)$ & $197(46)$ & 0.211 \\
\hline Two-step technique & $102(23)$ & $6(38)$ & $96(22)$ & 0.220 \\
\hline Use of capnography & $170(38)$ & $2(13)$ & $168(39)$ & 0.035 \\
\hline
\end{tabular}

ICU intensive care unit, MICU medical intensive care unit, SICU surgical intensive care unit, CCU cardiac intensive care unit, GCS Glasgow Coma Scale, RASS Richmond Agitation Sedation Scale 
developed complications used a mechanical ventilator $(14 / 16,88 \%)$ and an artificial airway $(15 / 16,94 \%)$ in our study. Assistant guidewires were used in both groups. Decreased or delirious mentality is a risk factor for respiratory malpositioning of a feeding tube $[2,16,17]$. Sedative and analgesic agents are used widely in critically ill patients because of pain, delirium, and agitation. Critically ill patients using a mechanical ventilator may exhibit a poor response due to sedation. These conditions are thought to be potential risk factors for tracheobronchial malpositioning of a feeding tube $[2,10,11]$. An artificial airway is actually a mechanical barrier; however, the low pressure cuffs of endotracheal and tracheal tubes do not represent a reliable barrier $[1,11]$. If an assistant guidewire is used, the small, flexible tube may slide into the trachea by the cuff along the tracheal wall [10]. Therefore, critically ill patients may be vulnerable to respiratory complications when inserting a tube.

Conventional methods of confirming tube position include auscultation of a bubbling sound in the epigastric region during air insufflation and aspiration of gastric fluid. However, these conventional methods are not useful and accurate to identify a malpositioned feeding tube [2-4]. Therefore, alternatively methods are required to verify feeding tube placement and prevent respiratory system complications. Chest X-ray is the gold standard to detect tracheal tube malpositioning $[9,11]$. This method confirms position after inserting the tube, but it is impossible to prevent respiratory malpositioning [9]. Thus, other methods to prevent a tube from entering the respiratory system are needed. Several approaches have been developed, such as fluoroscopic-, laryngoscopic-, and endoscopic-guided insertion. However, these techniques increase cost and time and require a specialist [2, 7]. Capnographic monitoring is a simple, cost-effective, and easily implemented. In addition, no specialist is needed for capnographic monitoring. Recent studies have reported advantages of bedside electromagnetic (EM)-guided feeding tube placement by specialized nurses [18-20]. Although EM-guided tube placement may help prevent respiratory complications while inserting a feeding tube, capnographic monitoring is more costeffective and simpler. Capnographic monitoring is useful to prevent respiratory malpositioning while inserting a feeding tube $[3,6,12,15,21]$.

Two respiratory complications cases were detected in the capnographic monitoring group. One patient was obese and was on a mechanical ventilator, making it difficult to insert a tube after repeated attempts. Another patient had used sedative drugs and mechanical ventilator. Tube insertion was difficult and subsequent attempts led to a pneumothorax. $\mathrm{CO}_{2}$ was repeatedly detected during capnographic monitoring but the respiratory complication could not be prevented.
This study had several limitations. This was a retrospective review of medical records at a single institution. A positive capnogram may not truly reflect malplacement of a feeding tube. No method was available to confirm false positives during capnographic monitoring. Delirium was defined when CAM-ICU was positive in this study. CAM-ICU is an objective and useful screening test to discriminate delirium. However, CAM-ICU cannot be performed when the patient is irritable or in deep sedative state $($ RASS $\leq 3)$. Sedative drugs were used when mechanical ventilator was applied or delirium was observed. Although sedative drugs were used when feeding tubes were inserted, adequate sedation may not have occurred in some patients. No comparison was made between times to place the feeding tubes between the groups. The individual characteristics and the skill of the physicians inserting the feeding tubes were not considered.

\section{Conclusions}

It is not rare for a small-bore feeding tube to be inserted incorrectly into the respiratory system in a critically ill patient. Respiratory complications decreased remarkably when capnographic monitoring was used while inserting a feeding tube. In addition, capnographic monitoring is a simple and easy to learn procedure, and no specialist is required. Our results reveal that capnographic monitoring is a simple and useful method to prevent respiratory complications while inserting a feeding tube in a critically ill patient.

\footnotetext{
Abbreviations

ARDS: Acute respiratory distress syndrome; CAM-ICU: Confusion assessment method for the intensive care unit; $\mathrm{CO}_{2}$ : Carbon dioxide;

EM: Electromagnetic; $\mathrm{ETCO}_{2}$ : End tidal carbon dioxide; GCS: Glasgow Coma Scale; ICU: Intensive care unit; IQR: Interquartile range; RASS: Richmond Agitation Sedation Scale
}

\section{Acknowledgements \\ There are no acknowledgments to add.}

Funding

Not applicable.

\section{Availability of data and materials}

As for the data availability, our data can be available on the Zenodo database (https://zenodo.org/record/44406\#.Vo3qeWDNuUk) as recommended repositories of BMC Anesthesiology.

\section{Authors' contributions}

JAR participated in study design, data collection, drafting of the manuscript, and the statistical analysis. KC participated in the statistical analysis and helped draft the manuscript. JHY participated in study design, performed the statistical analysis, and drafted the manuscript. DSL participated in the statistical analysis and helped draft the manuscript. GYS participated in study design and coordination and helped draft the manuscript. KJ participated in study design and drafting of the manuscript. JC participated in study conception and design, data collection, and drafting of the manuscript. CRC participated in study design and coordination. IS participated in the statistical analysis and helped draft the manuscript. KK participated in the statistical analysis and helped draft the manuscript. CMP participated in study conception and design, data collection, and drafting of the manuscript. All authors read and approved the final manuscript. 


\section{Competing interests}

The authors declare that they have no competing interests.

\section{Consent for publication}

Not applicable. This study did not contain any individual person's data in any form (including individual details, images or videos).

\section{Ethics approval and consent to participate}

This study was approved by the Institutional Review Board of Samsung Medical Center (SMC 2015-02-076-001) according to the Declaration of Helsinki on reviewing and publishing information from patient's records. Informed consent was waived because of the retrospective nature of the study.

\section{Author details}

'Department of Critical Care Medicine, Samsung Medical Center, Sungkyunkwan University School of Medicine, Seoul, Republic of Korea. ${ }^{2}$ Department of Surgery, Samsung Medical Center, Sungkyunkwan University School of Medicine, Seoul, Republic of Korea. ${ }^{3}$ Division of Cardiology, Department of Medicine, Samsung Medical Center, Sungkyunkwan University School of Medicine, Seoul, Republic of Korea. ${ }^{4}$ Division of Pulmonary and Critical Care Medicine, Department of Medicine, Samsung Medical Center, Sungkyunkwan University School of Medicine, Seoul, Korea. ${ }^{5}$ Research Institute for Future Medicine, Biostatistics and Clinical Epidemiology Center, Samsung Medical Center, Seoul, Korea.

Received: 31 October 2015 Accepted: 28 November 2016 Published online: 09 December 2016

\section{References}

1. Kolbitsch C, Pomaroli A, Lorenz I, Gassner M, Luger TJ. Pneumothorax following nasogastric feeding tube insertion in a tracheostomized patient after bilateral lung transplantation. Intensive Care Med. 1997:23:440-2

2. Rassias AJ, Ball PA, Corwin HL. A prospective study of tracheopulmonary complications associated with the placement of narrow-bore enteral feeding tubes. Crit Care. 1998;2:25-8.

3. Kindopp AS, Drover JW, Heyland DK. Capnography confirms correct feeding tube placement in intensive care unit patients. Can J Anaesth. 2001:48:705-10.

4. Fuchs J, Schummer C, Giesser J, Bayer O, Schummer W. Detection of tracheal malpositioning of nasogastric tubes using endotracheal cuff pressure measurement. Acta Anaesthesiol Scand. 2007;51:1245-9.

5. Boyes RJ, Kruse JA. Nasogastric and nasoenteric intubation. Crit Care Clin. 1992:8:865-78.

6. Munera-Seeley V, Ochoa JB, Brown N, Bayless A, Correia Ml, Bryk J, et al. Use of a colorimetric carbon dioxide sensor for nasoenteric feeding tube placement in critical care patients compared with clinical methods and radiography. Nutr Clin Pract. 2008;23:318-21.

7. de Aguilar-Nascimento JE, Kudsk KA. Clinical costs of feeding tube placement. JPEN J Parenter Enteral Nutr. 2007;31:269-73.

8. de Aguilar-Nascimento JE, Kudsk KA. Use of small-bore feeding tubes: successes and failures. Curr Opin Clin Nutr Metab Care. 2007;10:291-6.

9. Ryu JA, Cho J, Park SB, Lee D, Chung CR, Yang JH, et al. Respiratory complications associated with insertion of small-bore feeding tube in critically ill patients. Korean J Crit Care Med. 2014;29:131-6.

10. Odocha O, Lowery Jr RC, Mezghebe HM, Siram SM, Warner OG. Tracheopleuropulmonary injuries following enteral tube insertion. J Natl Med Assoc. 1989;81:275-81

11. Luo RB, Zhang M, Gan JX. Tracheobronchial malposition of fine bore feeding tube in patients with mechanical ventilation. Crit Care. 2011:15:454

12. Meyer P, Henry M, Maury E, Baudel JL, Guidet B, Offenstadt G. Colorimetric capnography to ensure correct nasogastric tube position. J Crit Care. 2009; 24:231-5.

13. Chau JP, Lo SH, Thompson DR, Fernandez R, Griffiths R. Use of end-tidal carbon dioxide detection to determine correct placement of nasogastric tube: a meta-analysis. Int J Nurs Stud. 2011;48:513-21.

14. Galbois A, Vitry P, Ait-Oufella H, Baudel JL, Guidet B, Maury E, et al. Colorimetric capnography, a new procedure to ensure correct feeding tube placement in the intensive care unit: an evaluation of a local protocol. J Crit Care. 2011;26:411-4

15. Araujo-Preza CE, Melhado ME, Gutierrez FJ, Maniatis T, Castellano MA. Use of capnometry to verify feeding tube placement. Crit Care Med. 2002;30:2255-9.
16. Roubenoff R, Ravich WJ. Pneumothorax due to nasogastric feeding tubes. Report of four cases, review of the literature, and recommendations for prevention. Arch Intern Med. 1989;149:184-8.

17. Wendell GD, Lenchner GS, Promisloff RA. Pneumothorax complicating smallbore feeding tube placement. Arch Intern Med. 1991;151:599-602.

18. Gerritsen A, de Rooij T, van der Poel MJ, Dijkgraaf MG, Bemelman WA, Busch OR, Besselink MG, Mathus-Vliegen EM. Endoscopic versus bedside electromagnetic-guided placement of nasoenteral feeding tubes in surgical patients. J Gastrointest Surg. 2014;18:1664-72.

19. Windle EM, Beddow D, Hall E, Wright J, Sundar N. Implementation of an electromagnetic imaging system to facilitate nasogastric and post-pyloric feeding tube placement in patients with and without critical illness. J Hum Nutr Diet. 2010;23:61-8.

20. Hemington-Gorse SJ, Sheppard NN, Martin R, Shelley O, Philp B, Dziewulski $P$. The use of the Cortrak Enteral Access System for post-pyloric (PP) feeding tube placement in a Burns Intensive Care Unit. Burns. 2011;37:277-80.

21. Burns SM, Carpenter R, Truwit JD. Report on the development of a procedure to prevent placement of feeding tubes into the lungs using endtidal CO2 measurements. Crit Care Med. 2001;29:936-9.

\section{Submit your next manuscript to BioMed Central and we will help you at every step:}

- We accept pre-submission inquiries

- Our selector tool helps you to find the most relevant journal

- We provide round the clock customer support

- Convenient online submission

- Thorough peer review

- Inclusion in PubMed and all major indexing services

- Maximum visibility for your research

Submit your manuscript at www.biomedcentral.com/submit
Biomed Central 